by Kazuhei Kikuchi $^{{ }^{*}}$, Kazutoshi Abiko ${ }^{2}$,Hiroyuki Nagahamal ${ }^{1}$ and Jun Muto ${ }^{1}$

\title{
Self-affinities analysis of fault-related folding
}

1 Department of Earth Science, Tohoku University, 6-3, Aramaki Aza Aoba, Aoba, Sendai, Miyagi 980-8578, Japan.

E-mail: kazuhei.kikuchi.s6@dc.tohoku.ac.jp

2 Industrial and Economic Department, Murayama Area General Branch Administration Office, Yamagata Prefectural Government, 19-68, 2 Chome, Teppomachi, Yamagata, Yamagata, Japan.

The Mid-Niigata Prefecture Earthquake did not rupture the earth's surface and occurred along a fault hidden well under folded terrain. In order to evaluate the seismic activity of such hidden earthquakes beneath fault-related folds, a new method to analyze self-affinities is introduced. Based on this analysis, the geometry of a fold is shown to be self-affine and can be variously scaled in different directions. The size and temporal properties, $b$-value in Gutenberg - Richter's law and p-value in the modified Omori's law, express aftershock activity. Then, from the analyzed data of the folds, we estimate the $b$ value and p-value of the hidden earthquake under the fault-related folds. These estimated $b$-values and $p$-values are concordant with seismologically obtained ones in the aftershock sequence of the Mid-Niigata Prefecture Earthquake.

\section{Introduction}

The Mid-Niigata Prefecture Earthquake occurred in Niigata Prefecture, Japan, $2004\left(M_{\mathrm{w}} 6.6\right)$. The main shock was followed by a large number of aftershocks with four being over $M_{\mathrm{w}} 6$ (Konagai et al., 2012). The Mid-Niigata Prefecture Earthquake took place in a fold-and-thrust belt that has been growing since the late Pliocene in a Miocene rift basin along the eastern margin of the Japan Sea (Okamura et al., 2007). The epicenter region of the Mid-Niigata Prefecture Earthquake is located near the Niigata-Kobe Tectonic Zone, recognized as a region of large strain rate along the Japan Sea coast and in the northern Chubu and Kinki districts of Japan (Sagiya et al., 2000). The focal mechanisms of these strong shocks, estimated by Hi-net and F-net (Honda et al., 2005) were reverse fault types oriented northwest that is concordant with the trend of pre-existing fold axes (Konagai et al., 2012). The epicenters of the aftershocks were distributed along the NNE and SSW direction within a length of about $30 \mathrm{~km}$ (Honda et al., 2005).

Okamura et al. (2007) earlier pointed out that the Mid-Niigata Prefecture Earthquake was associated with fault-related folding. Such earthquakes taken place in "blind" folded terrains are named "hidden earthquakes" (Stein and Yeats, 1989). The location and amount of slip for the hidden earthquakes cannot in fact be observed directly. The Northern Apennine Mountains in Italy have been built by earthquakes along faults hidden well under the surface. Such earthquakes unlike their more familiar counterparts do not rupture the earth's surface (Stein and Yeats, 1989).
The Mid-Niigata Prefecture Earthquake killed about 40 people and injured about 3000 , largely as a result of building collapse. More than 100,000 residents were forced to evacuate their homes (Sidle et al., 2005). Moreover, despite the moderate size of the earthquake, thousands of landslides caused damages to roads, farmland, or residential areas. The economic loss due to these landslides was initially estimated at 8 billion US dollars, making this one of the costliest landslide events in history (Kieffer et al., 2006). An effective and quick stabilization of slopes in the MidNiigata mountainous terrain is thus considered to be a pressing need (Konagai et al., 2012).

In general, many folds are apparently curved or jagged on a wide range of scales, so that their geometries appear to be similar when viewed at different magnifications. Using the method of Matsushita and Ouchi (1989a, 1989b), we analyzed the self-affinities of folds in the North Honshu Arc, Japan (Kikuchi et al., 2013). Nagumo (1969a, b) pointed out that the $b$-value of Gutenberg-Richter's law is related to the sharpness of plastic bending deformation of a medium. Under this Nagumo's assumption, Kikuchi et al. (2013) proposed a new relation between the $b$-value of Gutenberg-Richter's law and the selfaffinities of folds related to earthquakes. Moreover, Nanjo et al. (1998) derived a relation between the $p$-value of modified Omori's law and $b$-values. In this paper, we analyze the self-affinity for the folds existed near the epicenter of the Mid-Niigata Prefecture Earthquake. Then we estimate the $b$-value and $p$-value of the hidden earthquake under the fault-related folds.

\section{Data of the transect profiles of folds}

For folds in the inner belt of the Northeast Honshu Arc, Kikuchi et al. (2013) applied a method to examine the aftershock sequence along the transect profiles of folds shown by the lines 1 to 9 in Figure 2. In this study, we analyzed folds 8 and 9 included in the zone of the Niigata-Kobe Tectonic line.

Kikuchi et al. (2013) used the following method; first define the smallest fixed length scale as a unit length scale $\alpha$, and measure the curve length $N \alpha$ by this scale between arbitrary points $A$ and $B$ on the curve. Then, calculate the $x$ - and $y$-variances, $X^{2}$ and $Y^{2}$ of all measured points between the two points $A$ and $B$ :

$$
X^{2}=\frac{1}{N} \sum_{i=1}^{N}\left(x_{i}-x_{c}\right)^{2} \text { and } Y^{2}=\frac{1}{N} \sum_{i=1}^{N}\left(y_{i}-y_{c}\right)^{2},
$$

with

$$
x_{c}=\frac{1}{N} \sum_{i=1}^{N} x_{i} \text { and } y_{c}=\frac{1}{N} \sum_{i=1}^{N} y_{i}
$$

where $\left(x_{i}, y_{i}\right)$ is the coordinate of the $i$ th measured point $P_{i}$ on the 


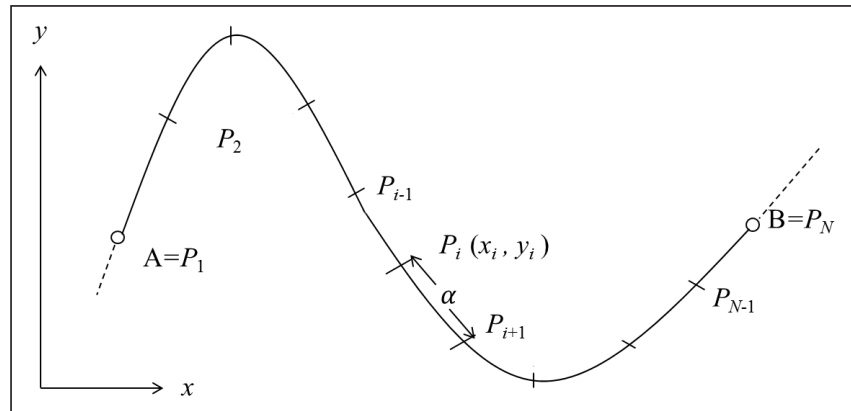

Figure 1. Measurement of a curve length $\mathrm{N}$ between a pair of points $A$ and $B$ on a given curve. $x$ and $y$ are coordinates, $\left(x_{i}, y_{i}\right)$ is the coordinate of the $i$-th measured point, $P_{i}\left(x_{i}, y_{i}\right)$ on the curve.

curve. The standard deviations of $X$ and $Y$ indicate the approximate widths of that part of the curve. Let us then repeat this measurement procedure for many pairs of points on the curve and determine the distribution using Log-Log plots of $X$ and $Y$ vs. $N$ whether they scale as

$$
X \propto N^{v_{x}}, Y \propto N^{v_{y}}
$$

where $v_{x}$ and $v_{y}$ are different in general.

The profiles near the epicentral region of the Mid Niigata Prefecture Earthquake (folds of the transect lines 8 and 9) are shown in Fig. 3a and Fig. 4a. From these profiles, $\log X-\log N$ and $\log Y$ Log $N$ can be approximated by straight lines (dotted lines) with different slopes $\left(v_{x} \neq v_{y}\right)$. This means that these profiles are differently scaled in a different direction indicating self-affine. The self-affine properties can be characterized by a Hurst exponent $\left(H=v_{y} / v_{x}\right)($ Feder,

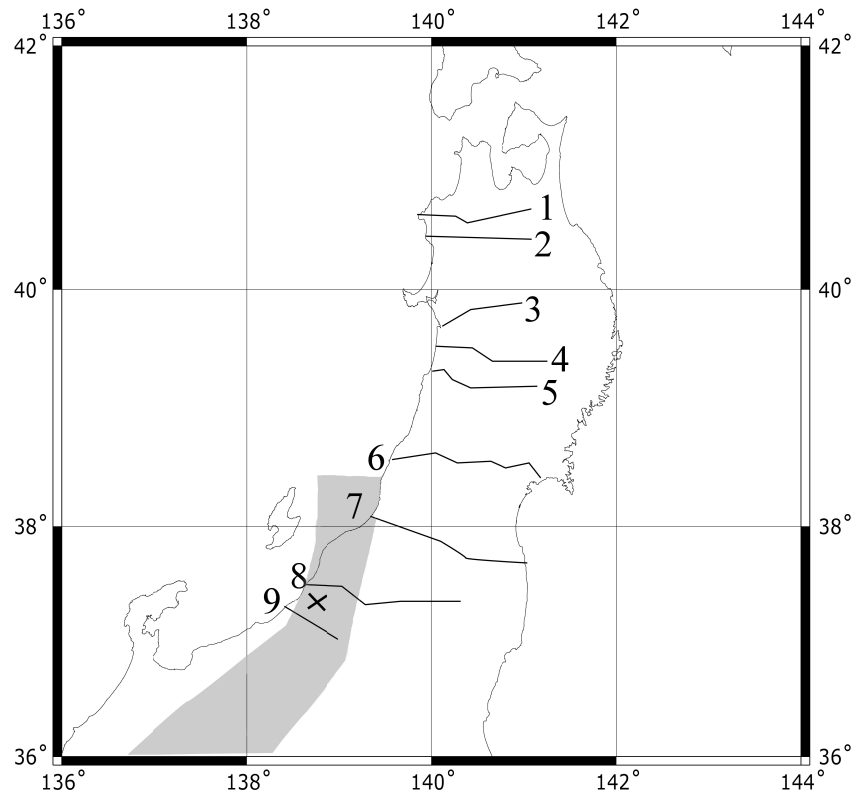

Figure 2. Map of the Northeast Honshu Arc. Numbered measurement lines are used for the analyses of self-affinity after Kitamura (1986). $\times$ represents epicenter of the Mid Niigata Prefecture Earthquake in $2004\left(M_{w} 6.6,37.3^{\circ} \mathrm{N}, 138.8^{\circ} \mathrm{E}\right)$. The Niigata-Kobe Tectonic Zone is denoted by a gray shaded area. Niigata-Kobe Tectonic Zone is recognized as a region of large strain rate along the Japan Sea coast and in the northern Chubu and Kinki districts, Japan (Sagiya et al., 2000).
1988; Peitgen and Saupe, 1988). From Fig. 3b, $H=0.84$ and $H=$ 0.88 from Fig. 4b. For a crossover from mid-Niigata area to Northeast Honshu Arc area, $\log Y$ - $\log N$ is approximated by two straight lines crossing at a point.

\section{Discussion}

Generally, the Fourier spectral density $\tilde{S}(k)$ of a scale-invariant curve is related to wave number $k$ by a power law equation. In this case the spectral exponent $\beta$ and the Hurst exponent $H$ are related as follows (Feder, 1988; Peitgen and Saupe, 1988) :

$$
\beta=2 H+1 \text {. }
$$

The $b$-value in the Gutenberg-Richter's law is widely used as a measure of seismicity. Nagumo (1969a, b) proposed a relationship between the spectral exponent $\beta$ and the $b$-value of Gutenberg-
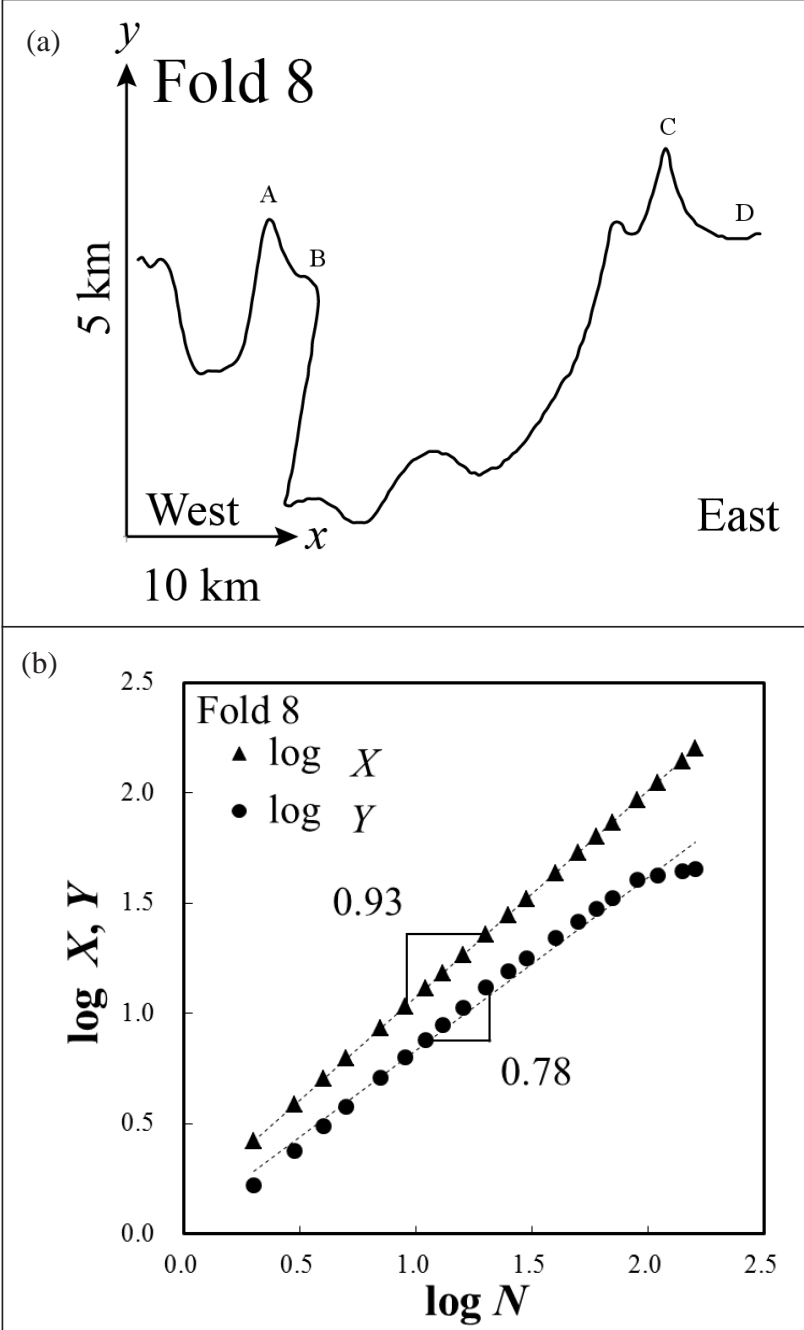

Figure 3. Analysis of the self-affinity of Fold 8. (a) Transect profile of Fold 8. Oginojo anticline (A), Yakushitoge pass (B), Higasiyama anticline $(C)$ and the vicinity of Muikamachi fault (D). Muikamachi fault is the source fault of the Mid Niigata Prefecture Earthquake. (b) Log-Log plot of horizontal and vertical standard deviations (X and $Y)$ and curve length $N$. Pairs $(\log X, \log N)$ and $(\log Y, \log$ $N)$ are lineally approximated by the method of least square fitting. The slopes represent $v_{x} \approx 0.93, v_{y} \approx 0.78$ and $H=0.84$. 
Richter's law (Gutenberg and Richter, 1944; see Appendix I) given by

$$
\beta=6-4 b,
$$

under the following assumptions (see Appendix II).

Nagumo (1969a, b) pointed out that the $b$-value of GutenbergRichter's law is related to the sharpness of the plastic bending deformation of the medium. It was equated bending of the strata with fold. Under Nagumo's assumptions and Eqs. (3) and (4), Kikuchi et al. (2013) can derived a relation between the $b$-value of GutenbergRichter's law and the Hurst exponent $H$ for the crustal deformation as

$$
2 H=5-4 b .
$$

Based on Eq. (5) and the Hurst exponent $H$ of the fault-related folds, we can estimate $b$-values for the epicenter region of the Mid Niigata Prefecture Earthquake hidden below the fault-related fold. From the analyzed data of $H=0.84$ for Fold 8 and $H=0.88$ for Fold $9, b$-value can be estimated to be 0.83 and 0.81 , respectively. These estimated $b$-values are concordant with seismologically obtained
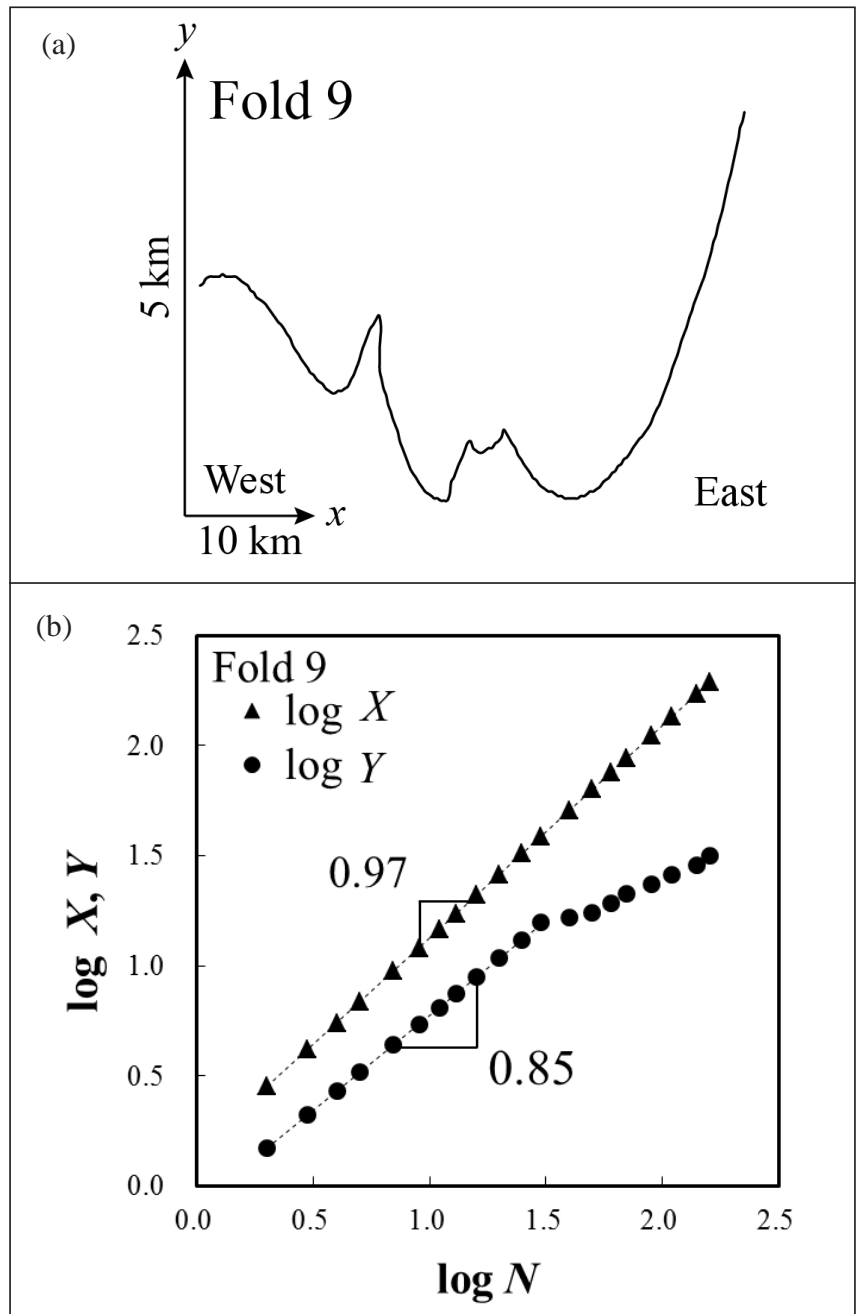

Figure 4. Analysis of the self-affinity of Fold 9. (a) Transect profile of Fold 9. (b) Log-Log plot of horizontal and vertical standard deviations $(X$ and $Y)$ and curve length $N$. Pairs $(\log X, \log N)$ and $(\log Y, \log N)$ are lineally approximated by the method of least square fitting. The slopes represent $v_{x} \approx 0.97, v_{y} \approx 0.85$ and $\mathrm{H}=0.88$. $b$-values of 0.80-0.87 in the aftershock sequence of the Mid Niigata Prefecture Earthquake (Enescu et al., 2007).

Nanjo et al. (1998) derived a relation between the $p$-value of the modified Omori's law (see Appendix III) and $b$-value by,

$$
b=(-2.10 \pm 0.35) p+(2.00 \pm 0.38)+\Delta,
$$

where $\Delta$ is a constant around $1.0 \sim 1.5$. In this paper, we use $\Delta=1.15$. The $p$-value in the modified Omori's law is widely used as a measure of seismicity. Based on Eq. (6) and the $b$-value of the faultrelated folds, we can estimate $p$-values for the epicenter region of the Mid Niigata Prefecture Earthquake hidden below the fault-related fold. From the analyzed data of $b=0.83$ for Fold 8 and $b=0.81$ for Fold $9, p$-value can be estimated to be 1.10 and 1.11 , respectively. These estimated $p$-values are concordant with seismologically obtained $p$-values of 1.10-1.13 in the aftershock sequence of the Mid Niigata Prefecture Earthquake (Enescu et al., 2007).

The size distribution of earthquakes expressed by the Gutenberg and Richter's law is equivalent to the fractal distribution of fault break areas by the relationship between the magnitude of the earthquake and its fault break area. The Hurst exponent for the crustal deformation can be related to the fractal dimension or the uniformity of the crustal fragmentation from mid-Niigata area to Northeast Honshu Arc area. Also, theoretical study suggested that $p$-value, which was a rate constant of aftershock decay, was related to the fractal dimension of a pre-existing fault system (Nanjo et al., 1998). Thus, we reinterpret the relationship between self-affinities of fold deformation and faultrelated folding. Hence, for the mitigation of natural disasters, we can evaluate statistical properties of seismicity ( $b$-value and $p$-value) of the hidden earthquakes by self-affinities of fault-related folds.

\section{Conclusion}

In order to evaluate the seismic activity of hidden earthquakes below the fault-related folds, a new method to analyze self-affinities is introduced. We applied it to large scale fold geometries of the Neogene and Paleogene around the epicenter region of the Mid Niigata Prefecture Earthquake. Based on this analysis, the geometry of the fold is shown to be self-affine and can be differently scaled in different directions. Based on the self-affinity of active folds, we can preestimate the $b$-values of the Gutenberg-Richter's law and $p$-value of the modified Omori's law for hidden earthquakes under the faultrelated folds. Therefore, this analysis method is useful to evaluate the risk of hidden earthquakes associated with fault-related folding as was discussed by Stein and Yeats (1989) in the Northern Apennine Mountains, Italy. We need to evaluate the future risk of hidden earthquakes which cause geohazards as landslides. In this paper, we have shown the importance of the geometrical structures (self-affinities) of active folding is a crucial one. In order to predict activities or aftershocks (i.e. to pre-estimate the $b$-values of the Gutenberg-Richter's law and $p$-values of the modified Omori's law) for hidden earthquakes occurred along a fault hidden well under folded terrain, an examination of the relation of the geometrical structures of pre-existing active fold systems will be an open challenge in the Earth Sciences.

\section{Acknowledgements}

The authors would like to thank Masataka Ando, Chien-Hsin Chang, Sumito Morita, Yasutaka Omori, Yasuji Sawada and an 
anonymous reviewer for valuable comments. K.K wishes to acknowledge Bhathiya Athurupana and Babu Ram Gyawali for help with the manuscript preparation. K.K. was supported by Tohoku University Institute for International Advanced Research and Education.

\section{References}

Enescu, B., J. Mori, and M. Miyazawa, 2007, Quantifying early aftershock activity of the 2004 mid-Niigata Prefecture earthquake $\left(\mathrm{M}_{\mathrm{w}} 6.6\right)$ : Journal of Geophysical Research, v. 112, B04310, DOI: 10.1029/2006JB004629.

Feder, J., 1988, Fractals: Plenum Press, New York.

Gutenberg, B., and C.F. Richter, 1944, Frequency of earthquakes in California: Bulletin of the Seismological Society of America, v. 34, pp. 185-188.

Honda, R., S. Aoi, N. Morikawa, H. Sekiguchi, T. Kunugi, and H. Fujiwara, 2005, Ground motion and rupture process of the 2004 Mid Niigata Prefecture earthquake obtained from strong motion data of K-NET and KiK-net: Earth, Planets and Space, v. 57, pp. 527-532.

Kieffer, S. D., R. Jibson, E. M. Rathje, and K. Kelson, 2006, Landslides triggered by the 2004 Niigata Ken Chuetsu, Japan, Earthquake: Earthquake Spectra, v. 22, pp. 47-73, DOI: 10.1193/1.2173021.

Kikuchi, K., K. Abiko, H. Nagahama, H. Kitazato, and J. Muto, 2013, Selfaffinities of landforms and folds in the Northeast Honshu Arc, Japan: Acta Geophysica, v. 61, pp. 1642-1658, DOI:10.2478/s11600-013-0151-z.

Kitamura, N. (ed.) 1986, Cenozoic Arc Terrane of Northeast Honshu, Japan: Hobundo, Sendai (in Japanese).

Konagai, K., Z. A. Kazmi, and Y. Zhao, 2012, Chapter 14 Extracting earthquake induced coherent soil mass movements: in S. D'Amico., ed, Earthquake Research and Analysis - New Frontiers in Seismology, In Tech, Shanghai, pp. 361-380, DOI: 10.5772/2458

Matsushita, M., and S. Ouchi, 1989a, On the self-affinity of various curves: Physica D, v. 38, pp. 246-251, DOI: 10.1016/0167-2789(89)90201-7.

Matsushita, M., and S. Ouchi, 1989b, On the self-affinity of various curves: Journal of the Physical Society of Japan, v. 58, pp. 1489-1492, DOI: 10.1143/JPSJ.58.1489.

Nagumo, S., 1969a, A derivation of Ishimoto-Iida's formula for the frequency distribution of earthquakes from a deformation fracture relation: Zisin (Seismological Society of Japan), v. 22, pp. 136-143 (in Japanese with English abstract).

Nagumo, S., 1969b, Deformation fracture relation in earthquake genesis and derivation of frequency distribution of earthquakes: Bulletin of the Earthquake Research Institute, The University of Tokyo, v.47, pp. 10151027.

Nanjo, K., H. Nagahama, and M. Satomura, 1998, Rates of aftershock decay and the fractal structure of active fault systems: Tectonophysics, v. 287, pp. 173-186.

Okamura, Y., T. Ishiyama, and Y. Yanagisawa, 2007, Fault-related folds above the source fault of the 2004 mid-Niigata Prefecture earthquake, in a foldand-thrust belt caused by basin inversion along the eastern margin of the Japan Sea: Journal of Geophysical Research, v. 112, B03S08, DOI: 10.1029/2006JB004320.

Omori, F. 1895, On the After-shocks of earthquakes: The Journal of the College of Science, Imperial University, Japan, v. 7, pp. 111-200.

Peitgen, H. O., and D. Saupe (eds.) 1988, The Science of Fractal Images, Springer, Berlin.

Sagiya, T., S. Miyazaki, and T. Tada, 2000, Continuous GPS array and presentday crustal deformation of Japan: Pure and Applied Geophysics, v. 157, pp. 2303-2322. DOI: 10.1007/PL00022507.

Sidle, C. R., T. Kamai, and A. C. Trandafir, 2005, Evaluating landslide damage during the 2004 Chuetsu Earthquake, Niigata Japan: EOS, v. 86, pp. 133-136.

Stein, R. and R. S. Yeats, 1989, Hidden earthquakes: Scientific American, v. 260, pp. 48-57.

Utsu, T. 1961, A statistical study on the occurrence of aftershocks: Geophysical Magazine, v. 30, pp.521-605.

\section{Appendix I: Gutenberg-Richter's low}

Gutenberg-Richter's law (Gutenberg and Richter, 1944) is expressed by

$$
\log N(M)=-b M-\phi,
$$

where $N(M)$ is the number of earthquakes with a magnitude greater than $M$, and $\phi$ and $b$ are constants.

\section{Appendix II: Nagumo's assumptions}

\section{Nagumo's assumption 1.}

The density of earthquake occurrence is assumed to be proportional to the curvature of the plastic bending deformation of the medium:

$$
N(x)=\frac{c}{d} \cdot\left|\frac{d^{2} w}{d x^{2}}\right|,
$$

where $N(x)$ is number of earthquakes, $c$ is a proportional constant, $d$ is an unit of dislocation, and $w$ is deflection.

Nagumo's assumption 2.

The frequency distribution of earthquakes with respect to their size is assumed to be proportional to the spectrum distribution of structural wave number of plastic deformation:

$$
N(a) d a=N(k) d k,
$$

where $a$ is an amplitude of seismic motion, and $k$ is wave number.

Nagumo's assumption 3.

The frequency distribution of earthquakes in a certain area is assumed to be proportional to the area of focal region:

$$
N(k)=S \cdot \int_{-\infty}^{\infty} N(x) e^{-i k x} d x
$$

where $S$ is an area of earthquake focal region.

\section{Appendix III: Modified Omori’s law}

The modified Omori's law (Utsu, 1961) is expressed by

$$
n(t)=\frac{\tilde{k}}{(\tilde{\mathrm{c}}+t)^{p}},
$$

where $n(t)$ is the frequency of aftershocks per unit time at time $t$ after the main shock, and $\tilde{k}, \tilde{\mathrm{c}}$, and $p$ are constants.

In the case of $p=1$, Equation (III-1) is given by

$$
n(t)=\frac{\tilde{k}}{(\tilde{\mathrm{c}}+t)},
$$

This relation is called Omori's law (1895). 